\title{
Comparison of Two DXA Systems, Hologic Horizon W and GE Lunar Prodigy, for Assessing Body Composition in Healthy Korean Adults
}

\author{
Seung Shin Park ${ }^{1}$, Soo Lim¹, Hoyoun Kim², Kyoung Min Kim³ \\ ${ }^{1}$ Department of Internal Medicine, Seoul National University Bundang Hospital, Seoul National University College of \\ Medicine; ${ }^{2}$ Biomedical Research Institute, Seoul National University Bundang Hospital, Seongnam; ${ }^{3}$ Division of Endocrinology, \\ Department of Internal Medicine, Yongin Severance Hospital, Yonsei University College of Medicine, Yongin, Korea
}

Background: Dual-energy X-ray absorptiometry (DXA) is the most widely used method for evaluating muscle masses. The aim of this study was to investigate the agreement between muscle mass values assessed by two different DXA systems.

Methods: Forty healthy participants ( 20 men, 20 women; age range, 23 to 71 years) were enrolled. Total and regional body compositional values for fat and lean masses were measured consecutively with two DXA machines, Hologic Horizon and GE Lunar Prodigy. Appendicular lean mass (ALM) was calculated as the sum of the lean mass of four limbs.

Results: In both sexes, the ALM values measured by the GE Lunar Prodigy ( $24.8 \pm 4.3 \mathrm{~kg}$ in men, $15.8 \pm 2.9 \mathrm{~kg}$ in women) were significantly higher than those assessed by Hologic Horizon $(23.0 \pm 4.0 \mathrm{~kg}$ in men, $14.8 \pm 3.2 \mathrm{~kg}$ in women). Furthermore, BMI values or body fat (\%), either extremely higher or lower levels, contributed greater differences between two systems. Bland-Altman analyses revealed a significant bias between ALM values assessed by the two systems. Linear regression analyses were performed to develop equations to adjust for systematic differences (men: Horizon ALM $[\mathrm{kg}]=0.915 \times$ Lunar Prodigy ALM $[\mathrm{kg}]+0.322, R^{2}=0.956$; women: Horizon ALM [kg]=1.066×Lunar Prodigy ALM [kg]-2.064, $R^{2}=0.952$ ).

Conclusion: Although measurements of body composition including muscle mass by the two DXA systems correlated strongly, significant differences were observed. Calibration equations should enable mutual conversion between different DXA systems.

Keywords: Absorptiometry, photon; Sarcopenia; Muscles

\section{INTRODUCTION}

Sarcopenia is a condition of frailty resulting from age-related loss of muscle mass and muscle function [1,2]. From the age of 60 years, skeletal muscle mass decreases by about $3 \%$ every

Received: 24 September 2021, Revised: 14 November 2021,

Accepted: 19 November 2021

Corresponding author: Kyoung Min Kim

Division of Endocrinology, Department of Internal Medicine, Yongin Severance Hospital, Yonsei University College of Medicine, 363 Dongbaekjukjeon-daero, Giheung-gu, Yongin 16995, Korea

Tel: +82-31-5189-8798, Fax: +82-31-5189-8567

E-mail: kyoungmin.kim@yonsei.ac.kr year, and it is known that individuals in their 80 s have a $30 \%$ to $50 \%$ reduction in skeletal muscle mass compared with that in their 40s [3]. Many biological changes related to aging contribute to this muscle loss, including declining hormone levels, denervation of muscle fibers, increased intracellular oxidative

\section{Copyright $\odot 2021$ Korean Endocrine Society}

This is an Open Access article distributed under the terms of the Creative Commons Attribution Non-Commercial License (https://creativecommons.org/ licenses/by-nc/4.0/) which permits unrestricted non-commercial use, distribution, and reproduction in any medium, provided the original work is properly cited. 
stress, and decreased caloric intake [4-7]. Because muscle plays diverse critical roles in human beings, sarcopenia is linked to various negative health outcomes, including falls, fractures, metabolic impairment, cardiovascular diseases and eventually, increased mortality [8-10]. Therefore, as the global population ages, sarcopenia is becoming an increasing important public health issue.

Sarcopenia is defined by low muscle mass accompanied by muscle weakness and/or impaired muscle function [1]. Therefore, diagnosis of sarcopenia requires documentation of low muscle mass plus either low muscle strength or low physical performance [11]. Therefore, accurate assessment of muscle mass is the most critical step in identifying sarcopenic individuals who have a high risk of negative health outcomes.

Several modalities have been used or studied to measure skeletal muscle mass, including bioelectrical impedance analysis (BIA), dual-energy X-ray absorptiometry (DXA), computed tomography (CT), and magnetic resonance imaging (MRI) [12]. $\mathrm{CT}$ and MRI are the gold standards for accurate measurement of muscle mass, but they are expensive and have limited accessibility [13]. BIA is highly accessible compared with CT or MRI, and also has benefits in terms of cost and radiation exposure risk. However, it has limited reliability because it can be greatly affected by the patient's hydration status and recent activity [14]. DXA is becoming the most widely used method to evaluate body composition parameters, including fat and lean mass, because of its superior accuracy and precision compared with BIA and its lower cost and radiation exposure risk compared with CT and MRI $[15,16]$.

Currently, several DXA machines are widely used; these include Hologic Horizon (Hologic Inc., Bedford, MA, USA), GE Lunar Prodigy (GE Healthcare Lunar, Madison, MI, USA), Norland (Norland Corp., Fort Atkinson, WI, USA). However, the measured values can differ significantly depending on the type of DXA machine used. In the case of bone mineral density (BMD) measurements, several conversion equations have been developed and used in clinical practice or research to allow data from different DXA machines to be merged or compared [1719]. However, conversion equations have not been developed for more recent applications of DXA, including body composition parameters such as lean mass. Therefore, standardizing muscle mass measured using different DXA machines remains difficult. In the present study, we analyzed the agreement between the muscle mass values assessed by the most widely used DXA machines, GE Lunar Prodigy and Hologic Horizon, in measuring body composition parameters and developed conver- sion equations to allow data obtained using these two systems to be compared. We also aimed whether any clinical parameters could affect greater differences between two DXA systems.

\section{METHODS}

\section{Participants}

The subjects were voluntarily included at the Seoul National University Bundang Hospital who agreed to undergo body compositional analysis using the two machines. We targeted the subjects with ages ranged from 20 to 70 years and their body mass index (BMI) from 15 to $40 \mathrm{~kg} / \mathrm{m}^{2}$. We categorized ages into five age groups with 10 -year intervals between 20 and 70 years old: younger than 30 years old, 30 to 39 years old, 40 to 49 years old, 50 to 59 years old, and older than 60 years. We also classified BMI as four categories with $5 \mathrm{~kg} / \mathrm{m}^{2}$ intervals between 15 and $40 \mathrm{~kg} / \mathrm{m}^{2}$ : lower than $20 \mathrm{~kg} / \mathrm{m}^{2}, 20$ to $25 \mathrm{~kg} / \mathrm{m}^{2}, 26$ to $30 \mathrm{~kg} / \mathrm{m}^{2}$, and over $30 \mathrm{~kg} / \mathrm{m}^{2}$. Then, we made a target enrollment table with five age groups and four BMI groups, totaling 20 categories of each age and BMI category, and tried to enroll at least one man and one woman in each category. Based on this enrollment target, we finally included 40 subjects (20 men and 20 women). Subjects who had a history of chronic or systemic diseases such as diabetes were excluded. Subjects with any medical histories that could affect their mobility and body composition, including osteoporosis and fracture, and, subjects having recent intentional weight loss or taking any medication that could affect body composition properties were also excluded. All participants provided informed consent. This study was approved by the Seoul National University Bundang Hospital Review Board (IRB No.: B-1704-390-007).

\section{Measurement of anthropometric and biochemical parameters}

Height and weight were measured to the nearest $0.1 \mathrm{~cm}$ and 0.1 $\mathrm{kg}$, respectively, with the participant in light clothing and not wearing shoes. Systolic and diastolic blood pressure was also measured. For biochemical analyses, blood samples were collected after overnight fasting of at least 8 hours and parameters including fasting glucose, insulin, lipid profiles, liver profiles, and renal function were assessed using standard protocols.

\section{DXA scans and data analysis}

For DXA assessments, all participants were given a light gown and pants to wear. Each participant was scanned consecutively on the same day, using both DXA machines, GE Lunar Prodigy 
DXA and Hologic Horizon W scanner according to the manufactures' standard protocols. The values for lean mass, fat mass, fat percent (\%), and BMD for both whole body and regional area were measured in both DXA machines. Scan mode selection was automatically processed by the manufacturer's software for GE Lunar Prodigy and array mode was used for Hologic Horizon W scanner following each manufacturer's protocol for body composition measures [20-22]. The results were analyzed using GE Lunar encore version 13.60 or Hologic APEX software 5.6.0.4. On both scanners, subjects were positioned with lateral hands position and $15^{\circ}$ internal position of both feet.

\section{Statistical analysis}

Each body composition measurement (total body fat mass, total body lean mass, trunk fat mass, trunk lean mass, and appendicular lean mass $[\mathrm{ALM}]$ ) by the two machines was analyzed using a paired $t$ test. Differences were considered significant when the $P$ value was $<0.05$. For each value, a linear regression model between the two machines was calculated. Each regression equation is shown as 'Hologic Horizon=slope $\times$ GE Lunar Prodigy+intercept.'

A Bland-Altman plot was used to compare the measurements by the two DXA systems. To verify the appropriateness of the conversion equation, we also calculated the regression parameters for the Hologic Horizon DXA based on the values obtained from the GE Lunar Prodigy DXA and the conversion equation, and compared these with the direct measurements by Hologic Horizon DXA using a Bland-Altman plot.

The chi-square test and Student's $t$ test were used to compare

Table 1. Baseline Characteristics of Study Subjects by Gender $(n=40)$

\begin{tabular}{|c|c|c|c|c|}
\hline Variable & Total $(n=40)$ & $\operatorname{Men}(n=20)$ & Women $(n=20)$ & $P$ value $^{\mathrm{a}}$ \\
\hline Age, yr & $42.8 \pm 13.8$ & $44.4 \pm 14.1$ & $41.2 \pm 13.7$ & 0.478 \\
\hline $\mathrm{SBP}, \mathrm{mm} \mathrm{Hg}$ & $126.5 \pm 15.9$ & $133.3 \pm 11.4$ & $119.6 \pm 17.0$ & 0.005 \\
\hline DBP, mm Hg & $74.8 \pm 10.7$ & $77.9 \pm 7.8$ & $71.8 \pm 12.4$ & 0.067 \\
\hline Weight, kg & $70.6 \pm 17.3$ & $78.9 \pm 14.8$ & $62.3 \pm 15.9$ & 0.002 \\
\hline Height, cm & $167.4 \pm 10.1$ & $175.5 \pm 5.8$ & $159.4 \pm 6.4$ & $<0.001$ \\
\hline BMI, $\mathrm{kg} / \mathrm{m}^{2}$ & $25.1 \pm 5.5$ & $25.6 \pm 4.5$ & $24.6 \pm 6.4$ & 0.194 \\
\hline $\mathrm{HbA1c}, \%$ & $5.5 \pm 0.3$ & $5.5 \pm 0.4$ & $5.4 \pm 0.3$ & 0.105 \\
\hline Insulin, $\mu \mathrm{IU} / \mathrm{mL}$ & $8.8 \pm 4.2$ & $8.4 \pm 3.3$ & $9.3 \pm 5.0$ & 0.495 \\
\hline Total cholesterol, mg/dL & $187.8 \pm 36.4$ & $177.5 \pm 28.1$ & $198.2 \pm 41.3$ & 0.072 \\
\hline Triglyceride, mg/dL & $96.8 \pm 49.7$ & $98.7 \pm 52.3$ & $95.0 \pm 48.2$ & 0.820 \\
\hline LDL-C, mg/dL & $104.3 \pm 27.6$ & $101.8 \pm 26.4$ & $106.8 \pm 29.2$ & 0.577 \\
\hline HDL-C, mg/dL & $57.3 \pm 14.5$ & $52.3 \pm 12.1$ & $62.4 \pm 15.3$ & 0.027 \\
\hline AST, IU/L & $22.4 \pm 5.4$ & $22.0 \pm 4.7$ & $22.9 \pm 6.0$ & 0.623 \\
\hline ALT, IU/L & $21.9 \pm 9.6$ & $25.1 \pm 9.8$ & $18.6 \pm 8.4$ & 0.030 \\
\hline Calcium, mg/dL & $9.4 \pm 0.4$ & $9.5 \pm 0.3$ & $9.3 \pm 0.4$ & 0.148 \\
\hline Phosphate, mg/dL & $3.6 \pm 0.4$ & $3.5 \pm 0.4$ & $3.8 \pm 0.4$ & 0.034 \\
\hline Total protein, $\mathrm{g} / \mathrm{dL}$ & $7.4 \pm 0.3$ & $7.4 \pm 0.4$ & $7.3 \pm 0.3$ & 0.291 \\
\hline Albumin, g/dL & $4.5 \pm 0.2$ & $4.6 \pm 0.2$ & $4.5 \pm 0.2$ & 0.025 \\
\hline Total bilirubin, mg/dL & $0.9 \pm 0.4$ & $1.0 \pm 0.4$ & $0.8 \pm 0.3$ & 0.137 \\
\hline ALP, IU/L & $62.5 \pm 17.3$ & $62.0 \pm 9.2$ & $63.1 \pm 15.8$ & 0.837 \\
\hline $\mathrm{BUN}, \mathrm{mg} / \mathrm{dL}$ & $13.6 \pm 4.1$ & $14.2 \pm 4.5$ & $13.0 \pm 3.7$ & 0.345 \\
\hline Creatinine, mg/dL & $0.8 \pm 0.1$ & $0.9 \pm 0.1$ & $0.7 \pm 0.1$ & $<0.001$ \\
\hline
\end{tabular}

Values are expressed as mean \pm standard deviation.

SBP, systolic blood pressure; DBP, diastolic blood pressure; BMI, body mass index; HbA1c, hemoglobin A1c; LDL-C, low dense lipoprotein cholesterol; HDL-C, high dense lipoprotein cholesterol; AST, aspartate transaminase; ALT, alanine aminotransferase; ALP, alkaline phosphatase; BUN, blood urea nitrogen.

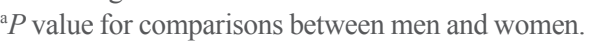


the baseline characteristics between the male and female participants. Pearson correlation analysis was used to identify whether the difference between the Hologic Horizon and GE Lunar Prodigy DXA measurements was correlated with other variables.

\section{RESULTS}

\section{Baseline characteristics of the study participants}

The baseline characteristics of the 40 participants (20 men, 20 women) are shown in Table 1. The mean age of the participants was 42.8 years and their mean glycated hemoglobin was 5.5\%. Mean weight, height and BMI were $70.6 \mathrm{~kg}, 167.4 \mathrm{~cm}$, and 25.1 $\mathrm{kg} / \mathrm{m}^{2}$, respectively. Systemic blood pressure, weight, height, high density lipoprotein, alanine transaminase and creatinine levels differed significantly between the male and female participants $(P=0.005, P=0.002, P<0.001, P=0.027, P=0.030$, and $P<0.001$, respectively), but the other parameters were similar between the sexes.

\section{Comparisons of body composition measurements by Hologic Horizon and GE Lunar Prodigy}

Generally, there was a very strong correlation between the measurements of body compositional parameters, both total and regional area, assessed by the GE Lunar Prodigy and Hologic Horizon (Fig. 1). However, significant differences were observed in several specific parameters. Table 2 shows the mean measures for all regional variables by the Hologic Horizon and GE Lunar Prodigy DXA: all values except trunk fat mass and trunk
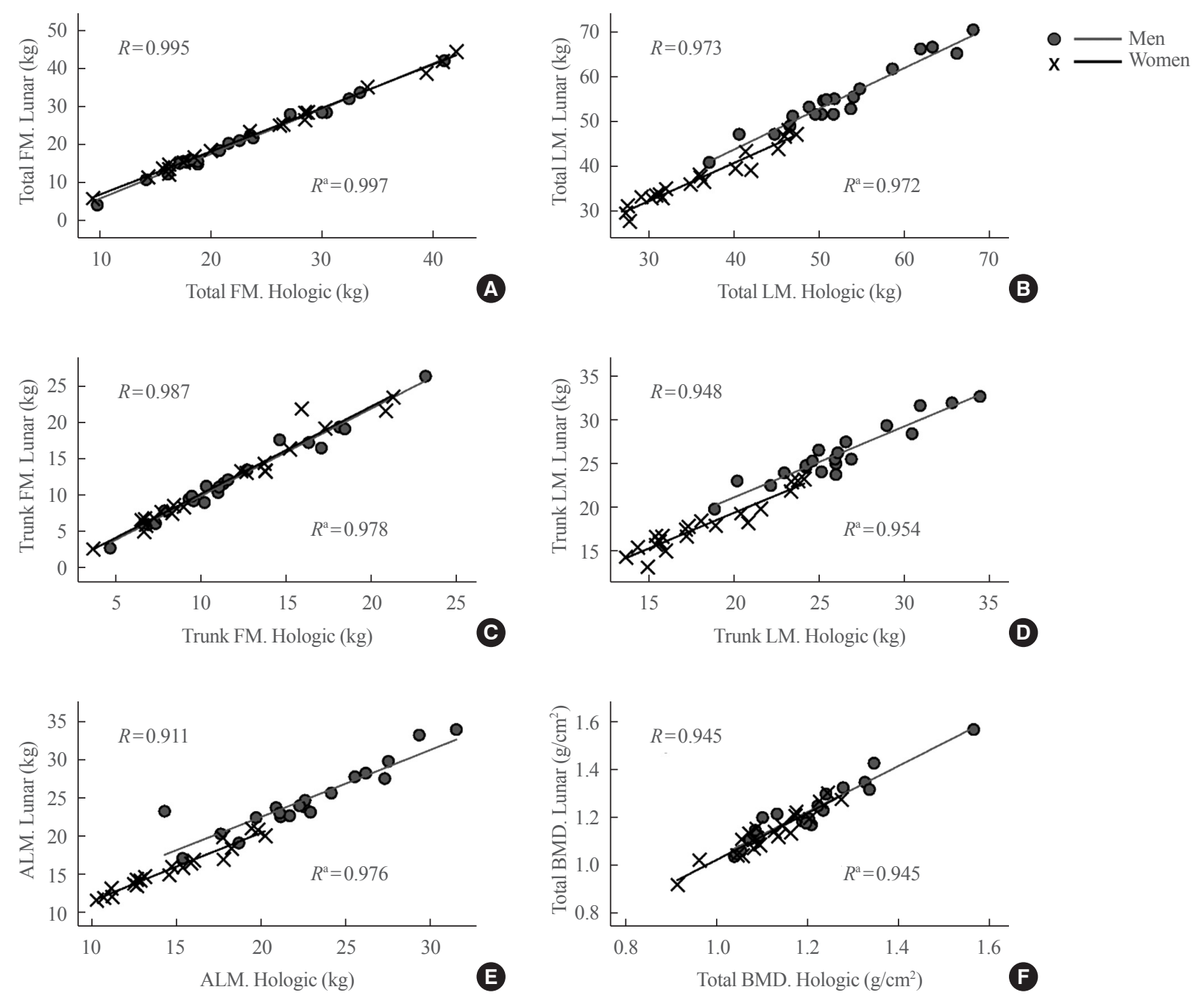

Fig. 1. Simple correlations between two systems, GE lunar and Hologic HORIZON for (A) total fat mass (FM), (B) total lean mass (LM), (C) trunk FM, (D) trunk LM, (E) appendicular lean mass (ALM), and (F) total bone mineral density (BMD). $R$, correlation coefficient for men. ${ }^{a} R$ for women. 
lean mass differed significantly between the two instruments. The values measured by the GE Lunar Prodigy for total body area, body mass composition, BMD and both total and regional lean mass were higher than those measured by the Hologic Horizon, whereas fat mass measured by the GE Lunar Prodigy was significantly lower than that measured by the Hologic Horizon (all $P<0.001$ in men; and $P=0.002, P=0.005$, and $P<0.001$ in women, respectively). The differences between the two instru- ments were greater in men than in women (Supplemental Fig. S1). Trunk fat mass and trunk lean mass values did not differ between the two DXA devices for either men or women.

The relationship between clinical parameters, anthropometric variables and DXA measures, and the differences between the two DXA devices, were analyzed to determine whether the differences between the Hologic Horizon and GE Lunar Prodigy DXA results were correlated with other clinical factors (Table 3 ).

Table 2. Comparisons of Values in Body Compositional Parameters between Hologic and Lunar in Men and Women

\begin{tabular}{|c|c|c|c|c|c|}
\hline Variable & Lunar & Hologic & $\begin{array}{l}\text { Absolute difference } \\
\text { (Lunar-Hologic) }\end{array}$ & $\%$ Differences & $P$ value \\
\hline \multicolumn{6}{|l|}{ Men } \\
\hline Total body area, $\mathrm{cm}^{2}$ & $2,591.2 \pm 286.4$ & $2,151.2 \pm 208.1$ & $440.0 \pm 110.5$ & $20.4 \pm 4.3$ & $<0.001$ \\
\hline Total body BMC, kg & $3.19 \pm 0.67$ & $2.60 \pm 0.51$ & $0.59 \pm 0.20$ & $22.4 \pm 5.6$ & $<0.001$ \\
\hline Total body BMD, $\mathrm{g} / \mathrm{cm}^{2}$ & $1.219 \pm 0.132$ & $1.199 \pm 0.131$ & $0.020 \pm 0.038$ & $1.7 \pm 3.2$ & 0.029 \\
\hline Total body fat, $\mathrm{kg}$ & $20.77 \pm 9.02$ & $22.75 \pm 7.63$ & $-1.98 \pm 1.60$ & $-12.0 \pm 13.4$ & $<0.001$ \\
\hline Total body lean, kg & $55.11 \pm 7.55$ & $52.50 \pm 8.01$ & $2.61 \pm 1.87$ & $5.3 \pm 4.1$ & $<0.001$ \\
\hline Trunk fat, kg & $12.19 \pm 5.63$ & $12.06 \pm 4.60$ & $0.12 \pm 1.33$ & $-2.5 \pm 15.5$ & 0.687 \\
\hline Trunk lean, kg & $26.07 \pm 3.33$ & $26.07 \pm 3.94$ & $-0.03 \pm 1.32$ & $0.4 \pm 5.4$ & 0.992 \\
\hline Rt. arm lean, kg & $3.22 \pm 0.66$ & $3.00 \pm 0.59$ & $0.23 \pm 0.16$ & $7.6 \pm 5.4$ & $<0.001$ \\
\hline Lt. arm lean, $\mathrm{kg}$ & $3.15 \pm 0.71$ & $2.86 \pm 0.58$ & $0.29 \pm 0.21$ & $9.9 \pm 6.4$ & $<0.001$ \\
\hline Rt. leg lean, kg & $9.29 \pm 1.55$ & $8.56 \pm 1.55$ & $0.73 \pm 0.44$ & $8.9 \pm 5.8$ & $<0.001$ \\
\hline Lt. leg lean, $\mathrm{kg}$ & $9.13 \pm 1.48$ & $8.59 \pm 1.41$ & $0.54 \pm 0.45$ & $6.6 \pm 5.7$ & $<0.001$ \\
\hline ALM, $\mathrm{kg}$ & $24.80 \pm 4.27$ & $23.01 \pm 4.00$ & $1.789 \pm 0.918$ & $7.9 \pm 4.1$ & $<0.001$ \\
\hline ALM/height, $\mathrm{kg} / \mathrm{m}^{2}$ & $8.03 \pm 1.22$ & $7.45 \pm 1.15$ & $0.58 \pm 0.29$ & $7.9 \pm 4.1$ & $<0.001$ \\
\hline ALM/weight, $\mathrm{kg} / \mathrm{kg}, \%$ & $31.7 \pm 3.5$ & $29.3 \pm 2.4$ & $0.02 \pm 0.01$ & $7.9 \pm 4.1$ & $<0.001$ \\
\hline ALM/BMI & $0.977 \pm 0.134$ & $0.904 \pm 0.104$ & $0.073 \pm 0.041$ & $7.9 \pm 4.1$ & $<0.001$ \\
\hline \multicolumn{6}{|l|}{ Women } \\
\hline Total body area, $\mathrm{cm}^{2}$ & $2,046.9 \pm 204.4$ & $1,763.2 \pm 139.6$ & $283.7 \pm 102.1$ & $16.0 \pm 5.3$ & $<0.001$ \\
\hline Total body BMC, kg & $2.33 \pm 0.40$ & $1.97 \pm 0.27$ & $0.36 \pm 0.17$ & $17.9 \pm 7.5$ & $<0.001$ \\
\hline Total body BMD, $\mathrm{g} / \mathrm{cm}^{2}$ & $1.134 \pm 0.094$ & $1.116 \pm 0.090$ & $0.017 \pm 0.031$ & $1.6 \pm 2.8$ & 0.020 \\
\hline Total body fat, $\mathrm{kg}$ & $22.64 \pm 10.92$ & $23.89 \pm 9.60$ & $-1.25 \pm 1.52$ & $-8.5 \pm 10.2$ & 0.002 \\
\hline Total body lean, $\mathrm{kg}$ & $37.18 \pm 5.96$ & $35.93 \pm 6.82$ & $1.25 \pm 1.74$ & $4.1 \pm 5.3$ & 0.005 \\
\hline Trunk fat, kg & $11.72 \pm 6.16$ & $11.59 \pm 5.31$ & $0.13 \pm 1.03$ & $-2.3 \pm 11.0$ & 0.586 \\
\hline Trunk lean, kg & $18.04 \pm 2.95$ & $18.23 \pm 3.47$ & $-0.19 \pm 1.09$ & $-0.4 \pm 6.3$ & 0.444 \\
\hline Rt. arm lean, kg & $1.83 \pm 0.39$ & $1.69 \pm 0.38$ & $0.13 \pm 0.09$ & $8.3 \pm 6.1$ & $<0.001$ \\
\hline Lt. arm lean, $\mathrm{kg}$ & $1.78 \pm 0.37$ & $1.58 \pm 0.38$ & $0.20 \pm 0.09$ & $13.6 \pm 6.6$ & $<0.001$ \\
\hline Rt. leg lean, kg & $6.20 \pm 1.13$ & $5.75 \pm 1.25$ & $0.45 \pm 0.32$ & $8.8 \pm 6.6$ & $<0.001$ \\
\hline Lt. leg lean, kg & $6.02 \pm 1.10$ & $5.79 \pm 1.27$ & $0.23 \pm 0.40$ & $5.0 \pm 7.4$ & 0.020 \\
\hline ALM, $\mathrm{kg}$ & $15.83 \pm 2.93$ & $14.82 \pm 3.20$ & $1.02 \pm 0.73$ & $7.7 \pm 5.7$ & $<0.001$ \\
\hline ALM/height, $\mathrm{kg} / \mathrm{m}^{2}$ & $6.23 \pm 1.08$ & $5.83 \pm 1.21$ & $0.40 \pm 0.28$ & $7.7 \pm 5.7$ & $<0.001$ \\
\hline ALM/weight, $\mathrm{kg} / \mathrm{kg}, \%$ & $26.0 \pm 3.4$ & $24.1 \pm 2.6$ & $0.02 \pm 0.01$ & $7.7 \pm 5.7$ & $<0.001$ \\
\hline ALM/BMI & $0.665 \pm 0.118$ & $0.617 \pm 0.097$ & $0.048 \pm 0.036$ & $7.7 \pm 5.7$ & $<0.001$ \\
\hline
\end{tabular}

Values are expressed as mean \pm standard deviation.

BMC, bone mineral contents; BMD, bone mineral density; Rt., right; Lt., left; ALM, appendicular lean mass; BMI, body mass index. 
Table 3. Associations of Baseline Clinical Parameters with Differences between Two DEX Machines

\begin{tabular}{|c|c|c|c|c|c|}
\hline Variable & Total body fat mass & Total lean mass & Trunk fat mass & Trunk lean mass & ALM \\
\hline \multicolumn{6}{|c|}{ Absolute difference (Lunar-Hologic) } \\
\hline Age, yr & $R=-0.179, P=0.270$ & $R=0.181, P=0.264$ & $R=-0.152, P=0.350$ & $R=0.269, P=0.093$ & $R=0.029, P=0.858$ \\
\hline Weight, $\mathrm{kg}$ & $R=0.537, P<0.001$ & $R=-0.391, P=0.013$ & $R=0.633, P<0.001$ & $R=-0.602, P<0.001$ & $R=-0.075, P=0.647$ \\
\hline Height, cm & $R=-0.195, P=0.228$ & $R=0.314, P=0.049$ & $R=0.032, P=0.846$ & $R=0.007, P=0966$ & $R=0.428, P=0.006$ \\
\hline BMI, $\mathrm{kg} / \mathrm{m}^{2}$ & $R=0.722, P<0.001$ & $R=-0.625, P<0.001$ & $R=0.696, P<0.001$ & $R=-0.680, P<0.001$ & $R=-0.337, P=0.033$ \\
\hline $\mathrm{HbA1c}, \%$ & $R=-0.026, P=0.872$ & $R=0.089, P=0.586$ & $R=-0.023, P=0.887$ & $R=0.103, P=0.527$ & $R=0.030, P=0.854$ \\
\hline Total cholesterol, mg/dL & $R=0.304, P=0.057$ & $R=-0.352, P=0.026$ & $R=0.200, P=0.215$ & $R=-0.428, P=0.006$ & $R=-0.021, P=0.898$ \\
\hline Triglyceride, $\mathrm{mg} / \mathrm{dL}$ & $R=0.331, P=0.037$ & $R=-0.332, P=0.036$ & $R=0.342, P=0.031$ & $R=-0.327, P=0.039$ & $R=-0.184, P=0.255$ \\
\hline LDL-C, mg/dL & $R=0.313, P=0.049$ & $R=-0.328, P=0.039$ & $R=0.244, P=0.130$ & $R=-0.482, P=0.002$ & $R=0.027, P=0.868$ \\
\hline HDL-C, mg/dL & $R=-0.132, P=0.418$ & $R=0.066, P=0.686$ & $R=-0.227, P=0.159$ & $R=0.158, P=0.331$ & $R=0.066, P=0.685$ \\
\hline AST, IU/L & $R=-0.161, P=0.321$ & $R=0.206, P=0.203$ & $R=-0.167, P=0.302$ & $R=0.243, P=0.130$ & $R=0.111, P=0.497$ \\
\hline ALT, IU/L & $R=-0.004, P=0.978$ & $R=0.107, P=0.513$ & $R=0.086, P=0.597$ & $R=-0.023, P=0.888$ & $R=0.168, P=0.299$ \\
\hline Total protein, g/dL & $R=0.094, P=0.563$ & $R=-0.023, P=0.887$ & $R=0.053, P=0.744$ & $R=-0.143, P=0.380$ & $R=0.105, P=0.520$ \\
\hline Albumin, g/dL & $R=-0.224, P=0.164$ & $R=0.248, P=0.123$ & $R=-0.164, P=0.312$ & $R=0.083, P=0.609$ & $R=0.320, P=0.044$ \\
\hline $\mathrm{BUN}, \mathrm{mg} / \mathrm{dL}$ & $R=-0.155, P=0.338$ & $R=0.212, P=0.189$ & $R=-0.172, P=0.288$ & $R=0.191, P=0.237$ & $R=0.151, P=0.351$ \\
\hline Creatinine, $\mathrm{mg} / \mathrm{dL}$ & $R=-0.230, P=0.153$ & $R=0.319, P=0.045$ & $R=-0.121, P=0.456$ & $R=0.004, P=0.983$ & $R=0.488, P=0.001$ \\
\hline Total body fat, $g$ & $R=0.830, P<0.001$ & $R=-0.782, P<0.001$ & $R=0.765, P<0.001$ & $R=-0.769, P<0.001$ & $R=-0.481, P=0.002$ \\
\hline Total body fat, $\%$ & $R=0.774, P<0.001$ & $R=-0.833, P<0.001$ & $R=0.565, P<0.001$ & $R=-0.627, P<0.001$ & $R=-0.642, P<0.001$ \\
\hline Total lean mass, $\mathrm{g}$ & $R=0.187, P=0.247$ & $R=-0.011, P=0.945$ & $R=0.374, P=0.017$ & $R=-0.332, P=0.036$ & $R=0.240, P=0.137$ \\
\hline ALM, kg & $R=0.145, P=0.373$ & $R=0.035, P=0.828$ & $R=0.327, P=0.039$ & $R=-0.303, P=0.058$ & $R=0.288, P=0.071$ \\
\hline \multicolumn{6}{|l|}{$\%$ difference (Lunar-Hologic) } \\
\hline Age, yr & $R=-0.146, P=0.367$ & $R=0.176, P=0.277$ & $R=-0.134, P=0.409$ & $R=0.218, P=0.177$ & $R=0.137, P=0.399$ \\
\hline Weight, $\mathrm{kg}$ & $R=0.576, P<0.001$ & $R=-0.618, P<0.001$ & $R=0.577, P<0.001$ & $R=-0.581, P<0.001$ & $R=-0.548, P<0.001$ \\
\hline Height, cm & $R=-0.089, P=0.584$ & $R=0.105, P=0.519$ & $R=0.038, P=0.816$ & $R=0.038, P=0.815$ & $R=0.006, P=0.969$ \\
\hline BMI, $\mathrm{kg} / \mathrm{m}^{2}$ & $R=0.707, P<0.001$ & $R=-0.776, P<0.001$ & $R=0.631, P<0.001$ & $R=-0.685, P<0.001$ & $R=-0.648, P<0.001$ \\
\hline $\mathrm{HbA1c,} \%$ & $R=-0.107, P=0.512$ & $R=0.003, P=0.983$ & $R=-0.105, P=0.519$ & $R=0.052, P=0.748$ & $R=-0.039, P=0.809$ \\
\hline Total cholesterol, mg/dL & $R=0.273, P=0.088$ & $R=-0.327, P=0.039$ & $R=0.187, P=0.247$ & $R=-0.472, P=0.002$ & $R=0.117, P=0.472$ \\
\hline Triglyceride, mg/dL & $R=0.345, P=0.029$ & $R=-0.337, P=0.033$ & $R=0.269, P=0.093$ & $R=-0.282, P=0.078$ & $R=-0.247, P=0.124$ \\
\hline LDL-C, mg/dL & $R=0.283, P=0.077$ & $R=-0.356, P=0.024$ & $R=0.206, P=0.202$ & $R=-0.501, P=0.001$ & $R=0.028, P=0.865$ \\
\hline HDL-C, mg/dL & $R=-0.087, P=0.591$ & $R=0.165, P=0.308$ & $R=-0.116, P=0.476$ & $R=0.087, P=0.593$ & $R=0.376, P=0.017$ \\
\hline AST, IU/L & $R=-0.138, P=0.395$ & $R=0.255, P=0.112$ & $R=-0.185 P=0.252$ & $R=0.237, P=0.141$ & $R=0.276, P=0.085$ \\
\hline ALT, IU/L & $R=-0.008, P=0.962$ & $R=0.025, P=0.880$ & $R=-0.038, P=0.817$ & $R=0.007, P=0.968$ & $R=0.041, P=0.800$ \\
\hline Total protein, g/dL & $R=0.176, P=0.278$ & $R=-0.057, P=0.729$ & $R=0.133, P=0.412$ & $R=-0.117, P=0.473$ & $R=0.014, P=0.930$ \\
\hline Albumin, g/dL & $R=-0.073, P=0.655$ & $R=0.205, P=0.204$ & $R=-0.049, P=0.762$ & $R=0.116, P=0.476$ & $R=0.220, P=0.173$ \\
\hline $\mathrm{BUN}, \mathrm{mg} / \mathrm{dL}$ & $R=-0.077, P=0.639$ & $R=0.183, P=0.258$ & $R=-0.062, P=0.706$ & $R=0.145, P=0.371$ & $R=0.188, P=0.245$ \\
\hline Creatinine, $\mathrm{mg} / \mathrm{dL}$ & $R=-0.093, P=0.570$ & $R=0.142, P=0.382$ & $R=-0.031, P=0.849$ & $R=0.003, P=0.987$ & $R=0.184, P=0.255$ \\
\hline Total body fat, $\mathrm{g}$ & $R=0.775, P<0.001$ & $R=-0.854, P<0.001$ & $R=0.664, P<0.001$ & $R=-0.756, P<0.001$ & $R=-0.682, P<0.001$ \\
\hline Total body fat, $\%$ & $R=0.706, P<0.001$ & $R=-0.732, P<0.001$ & $R=0.531, P<0.001$ & $R=-0.641, P<0.001$ & $R=-0.484, P=0.002$ \\
\hline Total lean mass, g & $R=0.285, P=0.074$ & $R=-0.290, P=0.069$ & $R=0.363, P=0.021$ & $R=-0.310, P=0.051$ & $R=-0.306, P=0.055$ \\
\hline ALM, kg & $R=0.259, P=0.107$ & $R=-0.249, P=0.121$ & $R=0.340, P=0.032$ & $R=-0.281, P=0.079$ & $R=-0.274, P=0.087$ \\
\hline
\end{tabular}

ALM, appendicular lean mass; BMI, body mass index; HbAlc, hemoglobin A1c; LDL-C, low dense lipoprotein cholesterol; HDL-C, high dense lipoprotein cholesterol; AST, aspartate transaminase; ALT, alanine aminotransferase; BUN, blood urea nitrogen. 

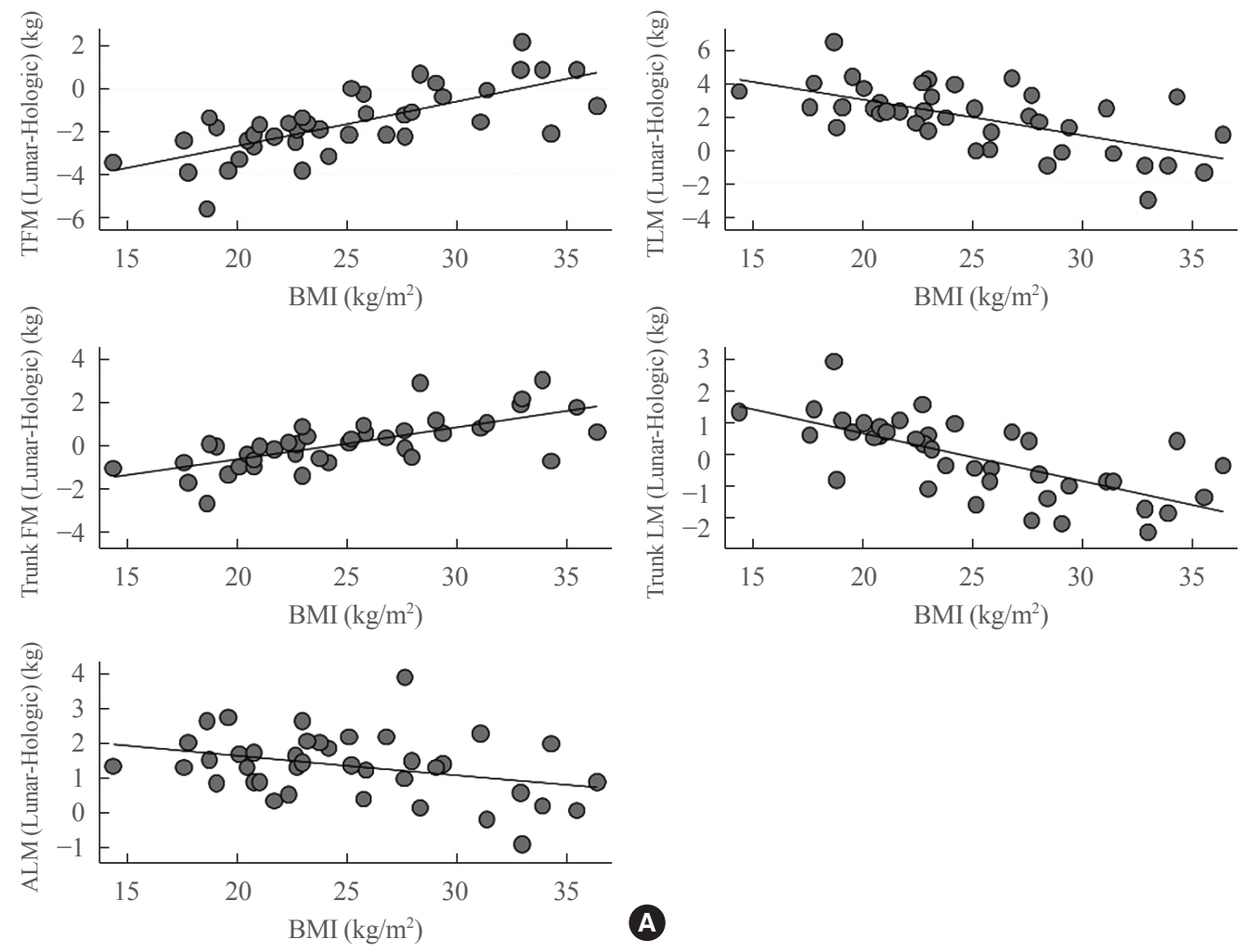

A
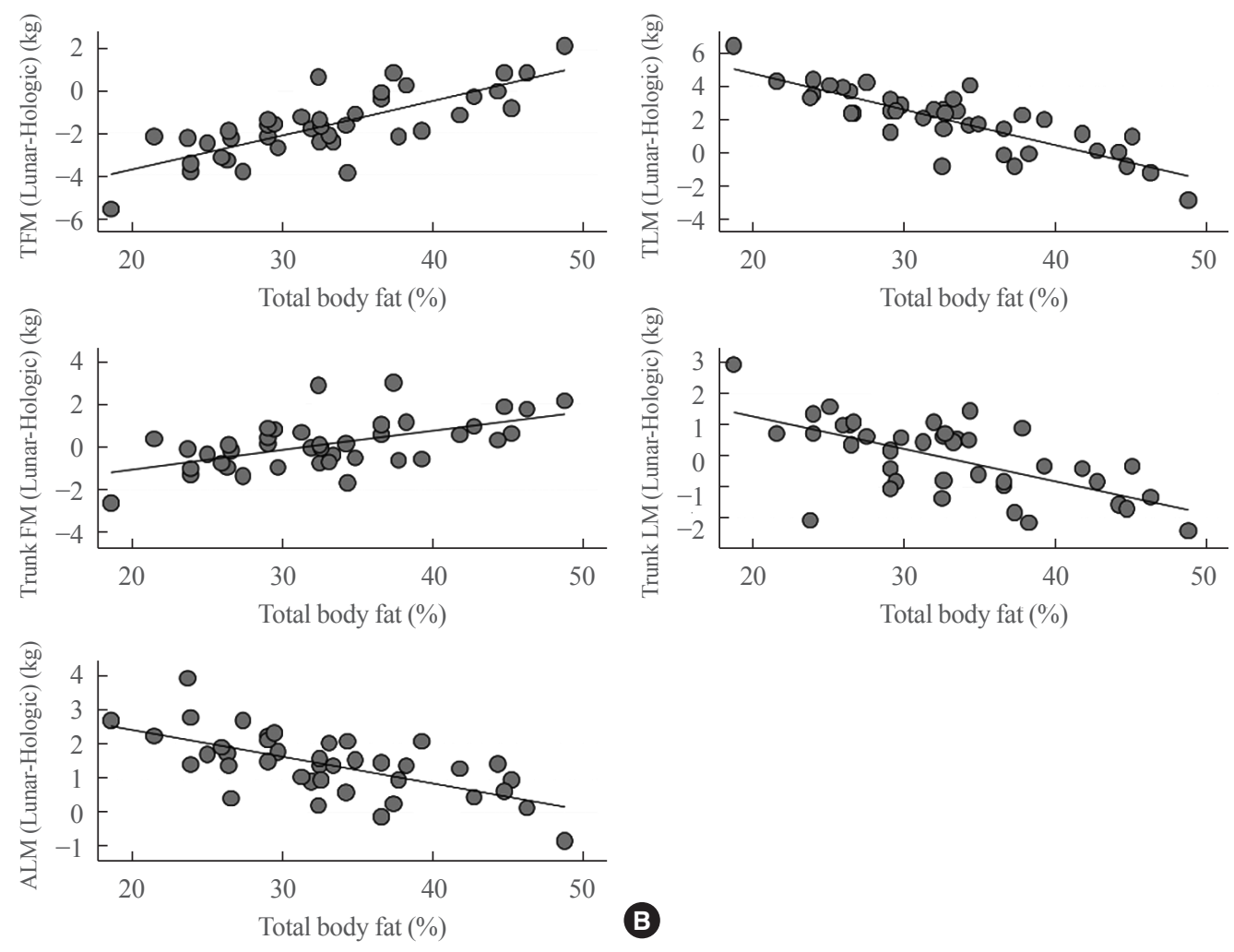

B

Fig. 2. Scatter plots of differences in total fat mass (TFM), total lean mass (TLM), trunk fat mass (FM), trunk lean mass (LM), and appendicular lean mass (ALM) values between GE Lunar and Hologic measurements according to (A) body mass index (BMI, $\left.\mathrm{kg} / \mathrm{m}^{2}\right)$ or $(\mathrm{B})$ total fat (\%). 
Both absolute and percent differences in total fat mass and lean mass, trunk fat mass and trunk lean mass and ALM were positively correlated with body weight, BMI, total body fat mass and total body fat $(\%)$. Moreover, the differences of trunk fat mass and lean mass were also positively correlated with total lean mass (Table 3). Furthermore, the levels of total cholesterol and low dense lipoprotein cholesterol (LDL-C) also provide consistent negative correlations with differences in lean mass values between two systems for both total and trunk lesions. The levels of triglyceride were also significantly correlated with fat or lean mass for both total and trunk lesions. However, these associations were not significant or were markedly attenuated after adjusting for total body fat percent (\%) (data not shown). The associations between BMI or body fat percent (\%) and the degree of difference between the two devices are plotted in Fig. 2 , and demonstrate that variance of the difference in total fat mass, total lean mass, trunk fat mass, trunk lean mass and ALM measures are greater when BMI is $<20$ or $>30 \mathrm{~kg} / \mathrm{m}^{2}$ (Fig. 2A). It showed similar pattern with body fat percent $(\%)$, and differences between two systems were greater in subjects with either higher or lower body fat percent (\%) (Fig. 2B).

\section{Development of equations to allow conversion of body compositional parameters between GE Lunar Prodigy and Hologic Horizon}

The equation 'Hologic Horizon=slope $\times$ GE Lunar Prodigy + intercept' was obtained by linear regression of total body fat mass, total body lean mass, trunk fat mass, trunk lean mass, and ALM values. The results of the linear regression are shown in Table 4. All five regression models were found to be statistically significant, and the $R^{2}$ values were all above 0.9 , except for the equation for trunk lean mass in men.

Fig. 3 shows the values produced by the two devices before and after the conversion. The differences in total body fat mass, total body lean mass, trunk fat mass and ALM assessed by the two DXA devices were $-1.98,2.61,0.12$, and 1.79 before conversion, respectively; all decreased to 0 after conversion using the regression equation. In the case of trunk lean mass, the difference decreased from 0.03 to 0.01 .

\section{DISCUSSION}

In the present study, we compared the body compositional values, especially muscle mass indices, measured by different two DXA systems, Hologic Horizon W and GE Lunar Prodigy, which are both widely used for measuring body composition parameters. Although a strong correlation was found between Hologic Horizon W and GE Lunar Prodigy DXA measurements, significant differences in fat mass and lean mass measurements were identified, with higher values for lean mass measures and lower values for fat mass measures with the GE Lunar Prodigy than with the Hologic Horizon W. Furthermore,

Table 4. Linear Regression Analyses for Total and Regional Body Fat or Lean Mass Measured GE Lunar and Hologic HORIZON

\begin{tabular}{|c|c|c|c|c|c|c|}
\hline Parameter & Regression equation & Slope $(P)$ & Intercept $(P)$ & $r^{2}$ & $P$ value & SEE \\
\hline \multicolumn{7}{|c|}{ Total body fat, $\mathrm{kg}$} \\
\hline Male & Hologic $=0.842 \times$ Lunar +5.263 & $<0.001$ & $<0.001$ & 0.991 & $<0.001$ & 0.75 \\
\hline Female & Hologic $=0.876 \times$ Lunar +4.046 & $<0.001$ & $<0.001$ & 0.995 & $<0.001$ & 0.72 \\
\hline \multicolumn{7}{|c|}{ Total body lean, $\mathrm{kg}$} \\
\hline Male & Hologic $=1.032 \times$ Lunar -4.392 & $<0.001$ & 0.190 & 0.946 & $<0.001$ & 1.91 \\
\hline Female & Hologic $=1.111 \times$ Lunar -5.391 & $<0.001$ & 0.037 & 0.944 & $<0.001$ & 1.65 \\
\hline \multicolumn{7}{|c|}{ Trunk fat mass, $\mathrm{kg}$} \\
\hline Male & Hologic $=0.805 \times$ Lunar +2.250 & $<0.001$ & $<0.001$ & 0.974 & $<0.001$ & 0.77 \\
\hline Female & Hologic $=0.858 \times$ Lunar +1.536 & $<0.001$ & $<0.001$ & 0.995 & $<0.001$ & 0.56 \\
\hline \multicolumn{7}{|c|}{ Trunk lean mass, $\mathrm{kg}$} \\
\hline Male & Hologic $=1.123 \times$ Lunar -3.194 & $<0.001$ & 0.188 & 0.899 & $<0.001$ & 1.29 \\
\hline Female & Hologic $=1.123 \times$ Lunar -2.030 & $<0.001$ & 0.194 & 0.912 & $<0.001$ & 1.06 \\
\hline \multicolumn{7}{|c|}{ Appendicular lean mass, $\mathrm{kg}$} \\
\hline Male & Hologic $=0.915 \times$ Lunar +0.322 & $<0.001$ & 0.786 & 0.956 & $<0.001$ & 0.87 \\
\hline Female & Hologic $=1.066 \times$ Lunar -2.064 & $<0.001$ & 0.036 & 0.952 & $<0.001$ & 0.72 \\
\hline
\end{tabular}



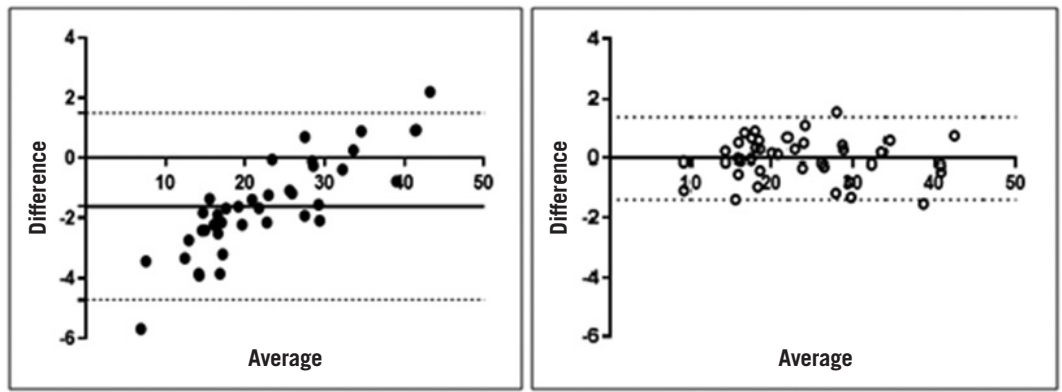

A
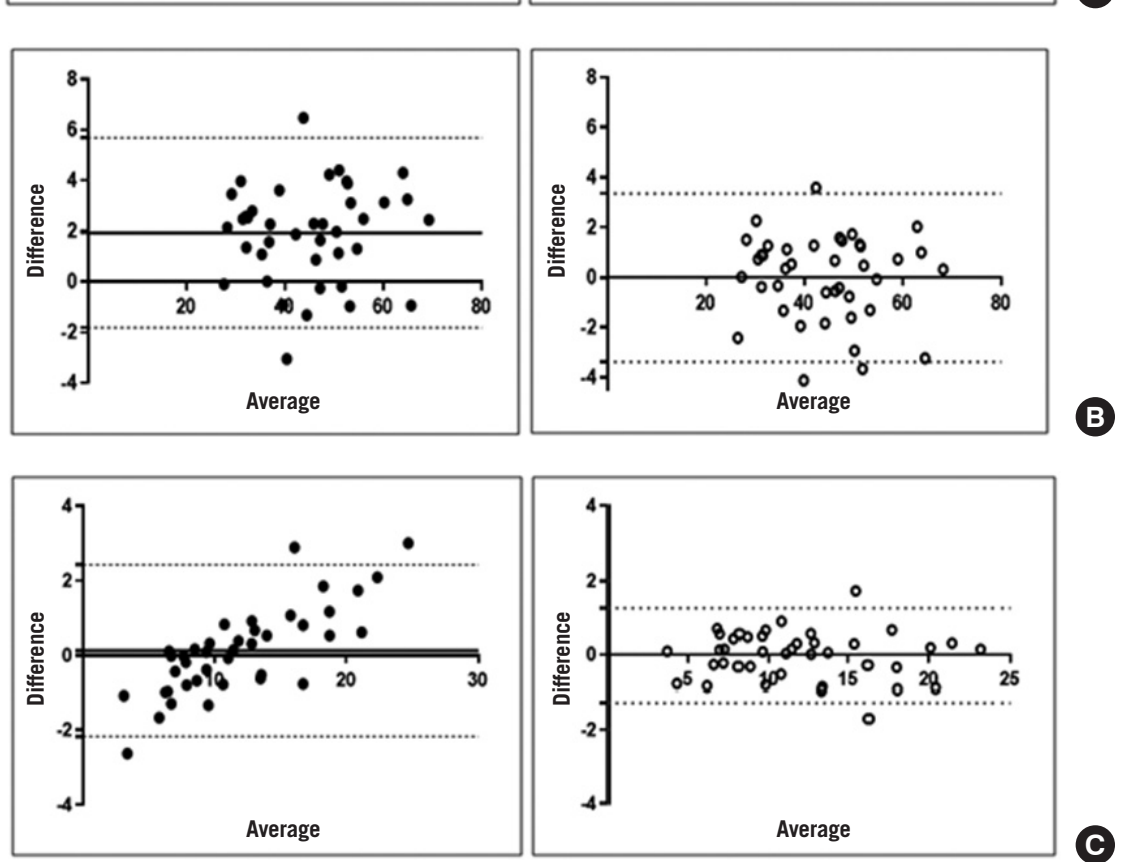

$\boldsymbol{C}$
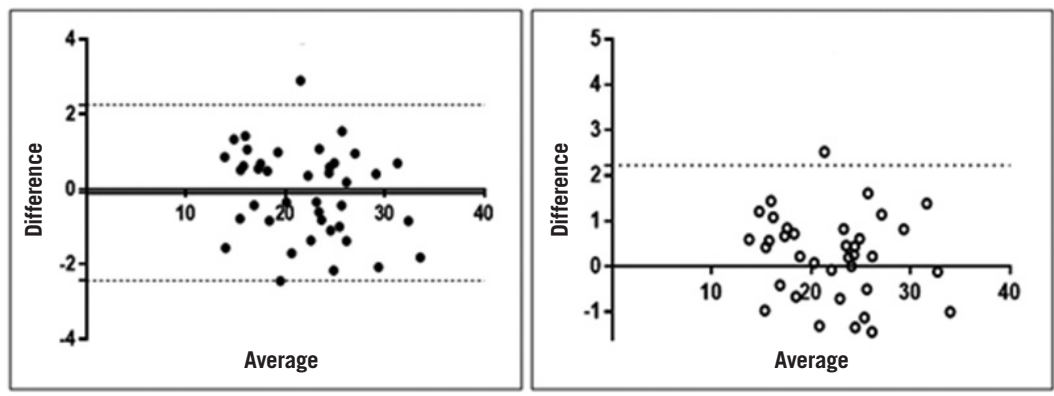

(D)
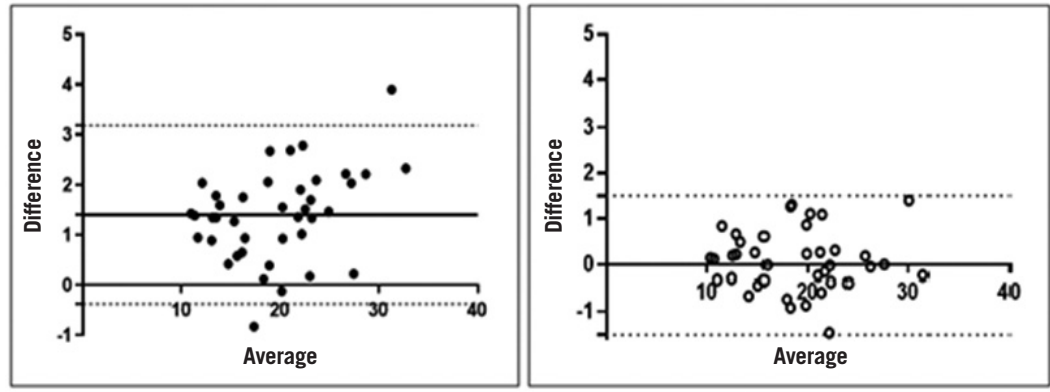

E

Fig. 3. Bland-Altman plot before and after adjustments for (A) total fat mass (TFM), (B) total lean mass (TLM), (C) trunk fat mass (FM), (D) trunk lean mass (LM), and (E) appendicular lean mass (ALM). 
obesity evaluated using BMI or body fat percent (\%), either extremely high or low, could affect the great variations between the two systems. From the present study, we developed conversion equations to convert GE Lunar Prodigy measurements to Hologic Horizon W values, and vice versa.

Sarcopenia, a syndrome of frailty driven by low muscle mass and impaired muscle function, is related to many adverse health outcomes, especially in older people. Therefore, it is of increasing concern because of the worldwide aging of society [23]. The accurate assessment of muscle mass in clinical practice is a crucial step in classifying sarcopenic individuals who are at high risk of adverse health outcomes. Among several modalities for measuring body composition, DXA is the most widely used because of its easy accessibility, low modality-related risks, reliability and lower cost. However, several different DXA systems are used for evaluating body composition parameters, which makes it difficult to compare each measurement accurately in research and clinical settings. For consistency in diagnosis of sarcopenia, a means to convert values obtained by different measuring systems is urgently needed.

Although strong correlations were generally observed between the values obtained by the two systems for both lean and fat masses in the present study, some significant differences were also seen. Values for whole-body fat mass and whole-body lean mass differed significantly between the two devices. Values for specific anatomic region-specific parameters, including arm lean mass, arm fat mass, leg lean mass, leg fat mass and ALM, also differed between the two devices. For measures of lean mass, including total lean mass and arm and leg lean mass, values obtained using the GE Lunar Prodigy were higher than those obtained using the Hologic Horizon. By contrast, measurements of fat mass using the Hologic Horizon gave higher values than those obtained using the GE Lunar Prodigy. However, values for trunk fat mass and trunk lean mass did not differ significantly between the two devices. This is consistent with previous studies using machines from the same manufacturers [24-26]. Body composition measurements using DXA are based on photon attenuation in target tissues according to tissue composition [27]. Although all DXA systems apply this common principle, differences between different systems can exist depending on the algorithms applied for selective tissue imaging, edge detection, region of interest definition and calibration methods [21].

The degree of dissimilarity between the two devices for measuring both lean mass and fat mass were significantly correlated with the participants' weight, total body fat (\%), and BMI. Fur- thermore, in terms of biochemical markers, particularly the lipid profiles, the levels of total cholesterol, LDL-C, and triglyceride, also provide significant associations with differences in values for both total and trunk between the two systems. Weight, BMI, total body fat $(\%)$, and lipid profiles are related to each other as fundamentally obesity-related profiles. Moreover, the observed associations between lipid profiles and the differences in body composition parameters between the two systems were altered or disappeared after adjusting for total body fat (\%). Therefore, it could suggest that obesity might be the main factor affecting the difference between the two devices, indicating variance of the difference in measurements by the two DXA machines may be greater if BMI or total body fat (\%) is extremely low or extremely high.

In the present study, the dissimilarities between measurements by the GE Lunar Prodigy and Hologic Horizon machines were compared between sexes. Sex disparity in conversion equations between the two DXA systems has been reported previously in evaluating BMD values [28]. In addition, body compositional properties are quite different according to sex for both quantitatively and proportionally throughout the growing and aging phases [29]. However, most of the previous reports of conversion equations between two different systems for body composition parameters were driven without considering the sex effect [5]. Therefore, we explored the sex effects in the differences between the two DXA systems and it turned out that significant differences were observed for total body lean mass and ALM, revealing it was greater in men than in women. The reason for this gender difference is not clear, but it may be because of differences in body composition between the genders. Therefore, we developed a linear equation for each gender to reflect these differences more accurately.

This study has several strengths. First, we enrolled participants with relatively wide ranges of BMI and age and attempted to produce equations that could be applied to various BMI and age groups. This wide BMI range in the study subjects may allow us to apply this equation to sarcopenic subjects with lower BMI or lean body mass. Second, healthy participants without any history of chronic diseases were enrolled to exclude the influence of different health conditions on body composition. Third, we also investigated which clinical and biochemical markers could significantly affect the differences between the two DXA systems and found that body fat (\%) could influence the large difference between two DXA machines. This information can be useful when comparing and interpreting body composition values measured by different DXA models in the same 
individual. Gender is another factor influencing the difference, so we provided a gender-specific conversion equation. Lastly, although a conversion equation between Hologic and GE Lunar was previously reported, different versions even from the same manufacturer could provide substantially different values [30]. Therefore, for the cross-calibration and merging of the data from the longitudinal studies or from widely varying systems, an exact conversion equation in each system is needed and we determined the conversion equation between Hologic Horizon $\mathrm{W}$ and GE Lunar Prodigy, where the most recent versions were used for assessing body composition. However, this study also had some limitations. First, none of the participants were children, and none weighed $<40 \mathrm{~kg}$. Previous studies have suggested that different equations are needed when the participant's weight is $<40 \mathrm{~kg}$. Therefore, the conversion equations used in the present study are unlikely to be applicable to children or extremely low-weight patients. Second, we did not compare our data with results from gold-standard techniques such as MRI. Comparisons with values from these modalities could help identify which devices are more accurate for measuring muscle mass and fat mass, and may allow the development of equations that convert the measurements by each instrument to a gold standard. Asian Working Group for Sarcopenia (AWGS) suggests cutoff values for low muscle mass [31], but does not mention the differences in the values measured from each DXA machines. Therefore, future updated guideline should further address this issue about the differences in body composition measures from different DXA systems and models to suggest absolute value when defining low muscle mass. Third, the numbers of study subjects were not calculated based on their statistical power, but were driven by previous reports. Finally, we did not compare the acquired values from the two DXA systems with those of BIA, which is another widely used modality for assessing body composition. However, taken together with the present conversion equations between the DXA systems and the previously reported calculation method between DXA and BIA [32], a further conversion from any of the DXA systems to BIA values could be applied.

Although the body composition measurements by two different DXA systems, Hologic Horizon and GE Lunar Prodigy, were generally consistent and correlated, significant differences were observed between the two systems. Body composition measurements for the trunk region were identical, but those by GE Lunar Prodigy for lean mass were significantly higher and those for fat mass were significantly lower compared with those by Hologic Horizon. We have established a linear equation to convert between values obtained by Hologic Horizon and GE Lunar Prodigy. This formula is expected to help provide consistent diagnosis of sarcopenic individuals with a higher risk of negative health outcomes.

\section{CONFLICTS OF INTEREST}

No potential conflict of interest relevant to this article was reported.

\section{ACKNOWLEDGMENTS}

All the study subject were enrolled and all the data were collected from the Seoul National University Bundang Hospital. This work was supported by the Korean Endocrine Society of Hyangseol Young Investigator Award 2018 to Kyoung Min Kim.

\section{AUTHOR CONTRIBUTIONS}

Conception or design: S.L., K.M.K. Acquisition, analysis, or interpretation of data: S.S.P., S.L., H.K., K.M.K. Drafting the work or revising: S.S.P., S.L., K.M.K. Final approval of the manuscript: S.S.P., S.L., H.K., K.M.K.

\section{ORCID}

Seung Shin Park https://orcid.org/0000-0001-5719-1539

Kyoung Min Kim https://orcid.org/0000-0001-8150-0266

\section{REFERENCES}

1. Goodpaster BH, Park SW, Harris TB, Kritchevsky SB, Nevitt M, Schwartz AV, et al. The loss of skeletal muscle strength, mass, and quality in older adults: the health, aging and body composition study. J Gerontol A Biol Sci Med Sci 2006;61:1059-64.

2. McGregor RA, Cameron-Smith D, Poppitt SD. It is not just muscle mass: a review of muscle quality, composition and metabolism during ageing as determinants of muscle function and mobility in later life. Longev Healthspan 2014;3:9.

3. Landi F, Cruz-Jentoft AJ, Liperoti R, Russo A, Giovannini S, Tosato M, et al. Sarcopenia and mortality risk in frail older persons aged 80 years and older: results from ilSIRENTE study. Age Ageing 2013;42:203-9.

4. Nieuwenhuizen WF, Weenen H, Rigby P, Hetherington MM. Older adults and patients in need of nutritional sup- 
port: review of current treatment options and factors influencing nutritional intake. Clin Nutr 2010;29:160-9.

5. Baumgartner RN, Waters DL, Gallagher D, Morley JE, Garry PJ. Predictors of skeletal muscle mass in elderly men and women. Mech Ageing Dev 1999;107:123-36.

6. Meng SJ, Yu LJ. Oxidative stress, molecular inflammation and sarcopenia. Int J Mol Sci 2010;11:1509-26.

7. Nilwik R, Snijders T, Leenders M, Groen BB, van Kranenburg J, Verdijk LB, et al. The decline in skeletal muscle mass with aging is mainly attributed to a reduction in type II muscle fiber size. Exp Gerontol 2013;48:492-8.

8. Cruz-Jentoft AJ, Landi F, Topinkova E, Michel JP. Understanding sarcopenia as a geriatric syndrome. Curr Opin Clin Nutr Metab Care 2010;13:1-7.

9. Yoo JI, Choi H, Ha YC. Mean hand grip strength and cut-off value for sarcopenia in Korean adults using KNHANES VI. J Korean Med Sci 2017;32:868-72.

10. Jin WS, Choi EJ, Lee SY, Bae EJ, Lee TH, Park J. Relationships among obesity, sarcopenia, and osteoarthritis in the elderly. J Obes Metab Syndr 2017;26:36-44.

11. Cruz-Jentoft AJ, Baeyens JP, Bauer JM, Boirie Y, Cederholm T, Landi F, et al. Sarcopenia: European consensus on definition and diagnosis. Report of the European Working Group on Sarcopenia in Older People. Age Ageing 2010;39:412-23.

12. Heymsfield SB, Gonzalez MC, Lu J, Jia G, Zheng J. Skeletal muscle mass and quality: evolution of modern measurement concepts in the context of sarcopenia. Proc Nutr Soc 2015;74:355-66.

13. Chien MY, Huang TY, Wu YT. Prevalence of sarcopenia estimated using a bioelectrical impedance analysis prediction equation in community-dwelling elderly people in Taiwan. $\mathrm{J}$ Am Geriatr Soc 2008;56:1710-5.

14. Sun G, French CR, Martin GR, Younghusband B, Green $\mathrm{RC}$, Xie YG, et al. Comparison of multifrequency bioelectrical impedance analysis with dual-energy X-ray absorptiometry for assessment of percentage body fat in a large, healthy population. Am J Clin Nutr 2005;81:74-8.

15. Bilsborough JC, Greenway K, Opar D, Livingstone S, Cordy J, Coutts AJ. The accuracy and precision of DXA for assessing body composition in team sport athletes. J Sports Sci 2014;32:1821-8.

16. Kim J, Wang Z, Heymsfield SB, Baumgartner RN, Gallagher D. Total-body skeletal muscle mass: estimation by a new dual-energy X-ray absorptiometry method. Am J Clin Nutr 2002;76:378-83.

17. Xu W, Chafi H, Guo B, Heymsfield SB, Murray KB, Zheng
J, et al. Quantitative comparison of 2 dual-energy X-ray absorptiometry systems in assessing body composition and bone mineral measurements. J Clin Densitom 2016;19:298304.

18. Lu Y, Fuerst T, Hui S, Genant HK. Standardization of bone mineral density at femoral neck, trochanter and Ward's triangle. Osteoporos Int 2001;12:438-44.

19. Hui SL, Gao S, Zhou XH, Johnston CC Jr, Lu Y, Gluer CC, et al. Universal standardization of bone density measurements: a method with optimal properties for calibration among several instruments. J Bone Miner Res 1997;12: 1463-70.

20. Hind K, Oldroyd B, Truscott JG. In vivo precision of the GE Lunar iDXA densitometer for the measurement of total body composition and fat distribution in adults. Eur J Clin Nutr 2011;65:140-2.

21. Oldroyd B, Treadgold L, Hind K. Cross calibration of the GE Prodigy and iDXA for the measurement of total and regional body composition in adults. J Clin Densitom 2018; 21:383-93.

22. Sutter T, Duboeuf F, Chapurlat R, Cortet B, Lespessailles E, Roux JP. DXA body composition corrective factors between Hologic Discovery models to conduct multicenter studies. Bone 2021;142:115683.

23. Buford TW. Sarcopenia: relocating the forest among the trees. Toxicol Pathol 2017;45:957-60.

24. Tothill P, Hannan WJ, Wilkinson S. Comparisons between a pencil beam and two fan beam dual energy X-ray absorptiometers used for measuring total body bone and soft tissue. Br J Radiol 2001;74:166-76.

25. Watson L, Venables M, Singh P, Murgatroyd P. An investigation into differences in body composition measurements between two GE Lunar densitometers and in comparison to a four component model. J Clin Densitom 2015;18:432-3.

26. Kaminsky LA, Ozemek C, Williams KL, Byun W. Precision of total and regional body fat estimates from dual-energy Xray absorptiometer measurements. J Nutr Health Aging 2014;18:591-4.

27. Plank LD. Dual-energy X-ray absorptiometry and body composition. Curr Opin Clin Nutr Metab Care 2005;8:305-9.

28. Ganda K, Nguyen TV, Pocock N. Gender disparity in BMD conversion: a comparison between Lunar and Hologic densitometers. Arch Osteoporos 2014;9:180.

29. Kim KM, Jang HC, Lim S. Differences among skeletal muscle mass indices derived from height-, weight-, and body mass index-adjusted models in assessing sarcopenia.

Copyright (C) 2021 Korean Endocrine Society 
Korean J Intern Med 2016;31:643-50.

30. Shepherd JA, Fan B, Lu Y, Wu XP, Wacker WK, Ergun DL, et al. A multinational study to develop universal standardization of whole-body bone density and composition using GE Healthcare Lunar and Hologic DXA systems. J Bone Miner Res 2012;27:2208-16.

31. Chen LK, Woo J, Assantachai P, Auyeung TW, Chou MY, Iijima K, et al. Asian Working Group for Sarcopenia: 2019 consensus update on sarcopenia diagnosis and treatment. J Am Med Dir Assoc 2020;21:300-7.

32. Lee SY, Ahn S, Kim YJ, Ji MJ, Kim KM, Choi SH, et al. Comparison between dual-energy X-ray absorptiometry and bioelectrical impedance analyses for accuracy in measuring whole body muscle mass and appendicular skeletal muscle mass. Nutrients 2018;10:738. 